\title{
Psychophysiological correlates of long-term attention to complex tasks
}

\author{
J. C. MILLER, N. Y. TAKAMOTO, G. M. BARTEL, and M. D. BROWN \\ U.S. Air Force School of Aerospace Medicine, Crew Technology Division (VN) \\ Brooks Air Force Base, Texas
}

\begin{abstract}
An ongoing investigation of the psychophysiological nature of attention is described. Problems associated with the measurement of long-term human attention to complex tasks are discussed. A multivariate, hierarchical regression approach is described for the inference, from the noninvasive measurement of surface neuroelectrical activities, of central nervous system operations associated with the maintenance and failure of long-term attention. The neuroelectrical signals used include the EEG, EOG, ECG, and impedance cardiogram. Psychophysiological measurement, data reduction, and analysis techniques are described.
\end{abstract}

Research in transportation safety strongly suggests that loss of vigilance is a common phenomenon in complex real-world operations (Mackie \& Miller, 1978). However, the extent of the loss has not been objectively determined (Miller \& Mackie, 1980). Vigilance research is the investigation of human behavioral and physiological responses in task settings characterized by the requirement to detect critical events ("signals," or "targets"), the occurrence of which may be very rare, but the early detection of which is vital to the effective or safe operation of a system with a human "in the loop."

Generally, investigations and field studies support the idea that humans perform poorly when asked to maintain vigilance for long periods of time. The performance decrement is subtle: Highly practiced psychomotor tasks continue to be performed at or near average, alerted baseline levels; however, intrusions of events of interest ("targets") that happen to be difficult to discriminate from background activities often go unnoticed. Such failures occur during periods called "mental lapses," or "microsleeps" (Williams, Lubin, \& Goodnow, 1959). Many tasks performed by humans have high demands for "vigilance." This type of monitoring is not restricted to classic watch-keeping tasks (radar, sonar, guard duty, etc.), but includes the day-to-day, month-to-month maintenance of attention to all kinds of repetitive tasks, including aircraft operations.

We are attempting to address that area between the classic laboratory vigilance task $-1 \mathrm{~h}$ of simple, successive discriminations at a high event rate (Miller \& Mackie, 1980) - and real-world day-to-day repetitive tasks, in order to generalize to the latter. To predict the occurrence of performance decrements in our task settings, we are studying several kinds of simultaneously occurring human neuroelectrical activities. The activities are viewed

Requests for reprints should be sent to James C. Miller, USAFSAM/VN, Brooks AFB, TX 78235-5000. in the context of hierarchies imposed by the functions of the neural structures involved in generating the neuro electrical signals of interest. This paper represents at overview of our test system.

\section{METHOD}

One of our objectives has been to develop tasks tha allow well-controlled behavioral assessments of long-tern attention. In terms of complexity, these tasks fall betwee simple laboratory tasks and simulated operational tasks Task selection was based upon several criteria. First, moderate degree of complexity was sought. Second, long-term attention component was required. Third, w looked for a history of statistical reliability. The Maniki Vigilance Task allows us to examine long-term attentio to a moderately complex spatial-processing demand. Th Multiple Task Performance Battery (MTPB) allows us t examine the performance of several simultaneou vigilance tasks and the effects on them of superimpose spatial or numeric processing tasks.

The Manikin task was "developed at the Royal Ai Force (RAF) Institute of Aviation Medicine, Farn borough, United Kingdom, as a complex reaction tim task that related to performance in modern system without being identified with any particular system (Reader, Benel, \& Rahe, 1981, p. 3). The RAF versio of the Manikin task is sensitive to hypoxia, alcohol, an other stressors. Additionally, performance of the task stable over time, and can be learned in 4 to 1025 -mi practice sessions (Reader et al., 1981). The little ma (manikin) in the CRT display varies in orientation, an pearing upright or inverted, and facing the viewer or fac ing away from the viewer. A cue, either a filled disk o square, is presented at the bottom of the display. Th viewer's task is to determine in which hand, left or righ the manikin holds a symbol matching the cue. The maniki always holds one disk in one hand and one square in th other. The set of options-upright or inverted, facing $\phi$ 
away, square or disk, and left or right-provides 16 display variations.

The sequence of display variations presented to the viewer in our implementation (PDP-11/34 system, Digital Equipment Corp., Maynard, MA; FORTRAN 77; RSX-11M) is fully random (Takamoto, 1983). Additionally, this implementation provides considerable control over many task parameters. The proportion of presentations within each of the four options may be specified by the investigator (e.g., $40 \%$ left, $60 \%$ right; $50 \%$ upright, $50 \%$ inverted; etc.). The time of display of the manikin may be set for any millisecond value up to $2,500 \mathrm{msec}$. The random interstimulus interval has a rectangular distribution from 3,000 to $12,000 \mathrm{msec}$. The viewer responds to each stimulus by pressing a "left" or a "right" button. The buttons are located under the index and middle fingers of one hand. The display blanks when a response is made. Mean reaction time, reaction time variance, and correct, incorrect, and omitted responses are recorded for each response, for each of the 16 manikin variation categories. We are particularly interested in correct, incorrect, and omitted target discriminations: The investigator sets one of the 16 manikin variations as the vigilance target. This target is displayed to the viewer for $10 \mathrm{sec}$ preceding task onset. Subsequent occurrences of the target require two pushes of the button by the viewer to indicate that the target has been perceived. Only a single button push is required for nontarget responding. Task length is set by the investigator; we use at least a 2 - $h$ uninterrupted task duration.

Software for the more complex task setting, the MTPB, has also been prepared for our use (Bartel, 1984). The development of the MTPB began in the 1950s at the Air Force Aerospace Medicine Research Laboratory (AFAMRL). Subsequently, work-rest cycles, sustained performance, and the effects of fever were extensively examined using this synthetic work task (Morgan \& Alluisi, 1972). The MTPB incorporates five individual tasks and one team task. They are Probability Monitoring, Blinking Light Monitoring, and Warning Light Monitoring, all vigilance tasks; Arithmetic Computation, a three-digit-number addition and subtraction task (logical/numeric processing); Target Identification, a spatialresolution task; and Code Lock Solving, a team-oriented logic task. Task performance is reliable and stable (Adams, Levine, \& Chiles, 1959). Our PDP-11/34 MTPB program (RSX-11M; FORTRAN 77) allows solo performances of the first five tasks. Each subject will work for an uninterrupted 2-h period, during which the three vigilance subtasks will run continuously and the arithmetic and spatial tasks will run discontinuously. (For example, during a 2.5 -h session of continuous vigilance performance, 30 min of arithmetic processing and, later, $30 \mathrm{~min}$ of spatial processing may be required.) Performance data are collected in 5-min epochs, including reaction times and variances and the numbers of correct, incorrect, and omitted responses for each subtask. We are particularly interested in correct, incorrect, and omitted target discriminations for the vigilance subtasks, and the degree to which higher cognitive demands interfere with the monitoring process.

Another objective has been to develop a multivariate method by which we can examine many neuroelectrical activities and relate them to measures of performance. For various technical reasons, we limit assessments to noninvasive electrophysiological measurement techniques. We are examining aspects of the electrical activities of the cerebral cortex (EEG), seventh cranial nerve (endogenous eye blinks), brainstem (ventilatory rate), sympathetic autonomic innervation of the ventricular myocardium (impedance cardiographic correlate of myocardial contractility), and parasympathetic autonomic innervation of the sinoatrial node (heart rate variability). The orientation of the investigations is to examine neural electrical activities associated with good and poor task performance, to reveal some of the central neural mechanisms underlying such behaviors. To accomplish these objectives, software has been prepared for the integrated processing of multiple channels of continuously recorded physiological data.

The task PHYDAT provides the ability to extract and condition digitized electrophysiological data for subsequent alignment with behavioral data, followed by multivariate statistical analyses. The data for PHYDAT are captured on analog tape in accordance with the InterRange Instrumentation Group (IRIG) B telemetry standards. The data are digitized (AD 11-K ADC) using a DEC PDP-11/34 system under RT-11 and stored on a 1,600-bpi magnetic tape file blocked at 2,048 bytes/ record. These data may consist of as many as 13 channels, each sampled 128 times/sec. A 2-h experiment may produce 30,000 blocks ( 512 bytes/record) of continuous data, segmented into $10-\mathrm{min}$ epochs. The size of the data set precludes implementation on the PDP-11/34; thus a VAX PDP-1 1/780 is used; an additional utility stores and converts the magnetic tape file to an RMS disk file, blocked at 512 bytes/record, for the VAX.

The programs running under PHYDAT, all of which were written in FORTRAN 77, may be categorized as Control, Query Interpretation, Data Description, Analysis Control, Data Acquisition, Raw Data Interpretation, and Ensemble Data Interpretation. Two raw data interpretation efforts are currently in use: impedance/electrocardjography and electrooculography. The first provides an initial scan of cardiovascular data for subsequent ensemble analysis. The second provides full data reduction for eyeblinks and saccadic eye movements. Frequency analysis of spontaneous activity and steady-state evoked potentials in the EEG will be added in the near future.

The cardiovascular data are generated by an electrocardiogram (ECG) amplifier, an impedance cardiograph, and a skin blood flow transducer. The IFM/Minnesota Impedance Cardiograph (ZCG; Instrumentation for Medicine, Inc., Greenwich, CT) sends a 4-mA, 100-kHz 
signal through the chest and measures the changing (resistive) impedance to that signal. The greatest change is due to breathing, but most of that slow oscillation is filtered out by the ZCG electronics. There remains a small pulsatile change in impedance (delta-Z) that has been shown to be related to the physical characteristics of the ejection of blood from the left ventricle of the heart into the aorta (Miller \& Horvath, 1978). To simplify calculations, it is the first derivative, $\mathrm{dz} / \mathrm{dt}$, of this signal that is processed. The $\mathrm{dz} / \mathrm{dt}$ signal for one cardiac cycle includes $\mathrm{B}, \mathrm{Z}$, and $\mathrm{X}$ waves, in that order. The $\mathrm{B}$ wave indicates atrioventricular valve closure (the beginning of the ejection of blood from the heart). The $Z$ wave indicates left ventricular ejection and the point of maximum rate of impedance change. The $\mathrm{X}$ wave indicates aortic valve closure (the end of ejection).

The other signal of interest is generated by an infrared detector (Beckman) clipped to the subject's earlobe. This device senses changes in skin blood flow, producing a pulse following each ventricular ejection. We are interested in the timing of the peak of that pulse (the "W wave"), with respect to the ECG R wave, since this interval gives an indication of the speed with which the pressure pulse associated with the ejection of blood from the heart reached the head (pulse transit time, or PTT). This speed is strongly affected by the rigidity of the conducting blood vessels; their rigidity is strongly related to blood pressure (Gribbin, Steptoe, \& Sleight, 1976; Steptoe, 1980).

The cardiovascular signals are sampled at 128 samples/sec. They are low-pass filtered $(3 \mathrm{~dB}$ down at $50 \mathrm{~Hz}$ ), and a $60-\mathrm{Hz}$ notch filter is used (Beckman signal conditioner). Baseline thoracic impedance is also recorded and sampled. The digital ECG trace is scanned in 2-sec increments for an R-wave occurrence, and then for a $T$-wave occurrence. The ZCG trace is scanned for the $Z$ wave, and then for the $B$ and $X$ waves. The interval between $B$ and $X$ establishes an estimate for the systolic ejection period (SEP). The skin blood flow trace is scanned for the peak corresponding with arrival of the pulse (W wave), and the R-to W-wave interval is ascertained. Descriptive statistics for R-R intervals and SEPs are calculated last. The variance associated with the $R-R$ intervals provides an estimate of heart rate variability.

The waveforms are ensemble averaged, using the ECG $R$-wave peak for synchronization, to enhance physical and electrical signal-to-noise ratios. The epoch length for sampling and analysis is $60 \mathrm{sec}$. The suggested approach was assembled from notes provided by $S$. $M$. Horvath and $R$. Marcus, Institute of Environmental Stress, University of California, Santa Barbara. The cardiovascular parameters discussed below are calculated from the ensembleaveraged impedance, ECT, and skin blood flow tracings. The Heather Index, a correlate of left ventricular myocardial contractility (Heather, 1969), is our principal indicator of autonomic sympathetic activity. The autonomic innervation of the ventricular myocardium is primarily sympathetic, allowing measures of myocardial con- tractility to provide indications of sympathetic activity that are relatively, although not completely, free of parasympathetic effects in our sedentary subjects. We hypothesize that sympathetic activity, manifested as the impedance correlate of left ventricular myocardial contractility, will be directly related to measures of performance in our longterm attention tasks. Conversely, due to the dominance of parasympathetic (over sympathetic) influence on the sinoatrial node in sedentary subjects, we hypothesize that heart rate variability (HRV) will be directly related to performance error rates. Additionally, the following calculated values are stored for analyses of cardiovascular activity: systolic ejection period, maximum height of $\mathrm{dz} / \mathrm{dt}$, preejection period, pulse transit time, chest fluid, stroke volume, stroke index, cardiac output, cardiac index, stroke work, total peripheral resistance, TPR index, Rwave amplitude, T-wave amplitude, $P-R$ interval, $R-T$ interval, and duration of electromechanical systole.

The endogenous eyeblink differs from its exogenous counterparts (reflex and voluntary blinks) by virtue of the lack of an identifiable, external eliciting stimulus (Stern, Walrath, \& Goldstein, 1984). The endogenous blink, measured by electrooculography, is of a form different from that of the voluntary blink. The voluntary and endogenous activities share the seventh (facial) cranial nerve for efferent innervation of the involved muscle, but different portions of the orbicularis palpebrarum muscle mediate the two blink processes: voluntary blinks, the septal and orbital portions; endogenous blinks, the pretarsal (connection to the tendo oculi) portion. The central signals triggering voluntary and endogenous blinks arise in the precentral cortex and travel via the corticobulbar tract to the cranial nerve nuclei.

Three observations allow the speculation that endogenous blinks may provide a window on the general level of activity in that portion of the midbrain reticular formation that participates in the diffuse, tonic activation of the rostral central nervous system: (1) the close physical relationship between the nucleus of the facial nerve and medullary structures involved in the sleep/wake process (n. reticularis pontis oralis, n. r. p. caudalis, $n$. giganto cellularis); (2) the suggestion that these midbrain reticular formation structures may integrate ocular activities to some degree (Bach-y-Rita \& Collins, 1971); and (3) the lack of identifiable external triggers for endogenous blinks. If true, one would expect quantifiable relationships between behavioral correlates of central neural activation and endogenous blink characteristics. In fact, investigations using both simulated real-world tasks and classic laboratory vigilance tasks have produced data suggesting that endogenous blink duration increases with time on task, whereas behavioral activation and target discrimination performance decline (Stern et al., 1984). The proportion of unusually long ( $>200 \mathrm{msec}$ ) blinks increased with time on task during simulated 20 -min automobile operations. Similarly, mean blink duration increased from 133 to $189 \mathrm{msec}$ from the first to the last $5 \mathrm{~min}$ spent in a 128-min discrimination task. During this 
latter investigation, it was also noted that the time-on-task effect held for auditory, as well as visual, discriminations, and that blink durations were slightly shorter for the visual than for the auditory task. Our hypothesis was that blink duration would increase as task performance, especially target discrimination, declined. The software needed to examine this hypothesis is in place, having been acquired from the Washington University (St. Louis, MO) Behavior Research Laboratory (J. A. Stern, personal communication, November 15, 1983). Summaries of blink durations and saccadic eye movement parameters for 1-min epochs are produced by the software.

The EOG raw data interpretation program assumes digitization at 100 sample/sec and 1,024 counts $/ V$, with endogenous blinks averaging about $500-\mathrm{mV}$ amplitude on a vertical-EOG trace on the IRIG B analog tape output, and saccades averaging about $20 \%$ of that size on a horizontal-EOG trace. The program first seeks a 32-count drop across one sample interval to signal the beginning of a possible endogenous blink. If the blink amplitude exceeds a subject-specific threshold and achieves a halfamplitude reopening with $500 \mathrm{msec}$, a blink is recorded. Currently, blinks exceeding $200 \mathrm{msec}$ at the halfamplitude point are classed as "long-duration" blinks, are counted separately from shorter blinks, and are not included in descriptive statistics for each 1-min epoch. Saccades are identified when $3 / 5$ of the subject-specific saccade criterion is exceeded across three sample intervals and $1 / 3$ of the criterion is exceeded across one of those intervals. These decision levels were established empirically by Stern and have worked well in our laboratory.

Ventilatory rate data are acquired from a thermistor placed below the subject's nostrils, registering temperature changes associated with inhalation and exhalation. The digitized ventilation trace for the entire experimental period is examined for inflection points. The reciprocal of the interval between end-inspiratory inflections is recorded as ventilation rate.

One of the great difficulties in the analysis of physiological data acquired from a small sample of subjects is that individual differences in physiologial responses (differing means and variances from subject to subject) tend to reduce the statistical confidence one has that particular effects are the result of an experimental manipulation. Additionally, comparisons of variables are difficult when the measures are expressed in different units. In order to "see" effects in our data when they are presented in the form of graphs or tables, and to reduce betweensubject differences for statistical purposes, we standardize the values for each measure, within subjects, before combining across subjects. The data are presented as standard scores ( $\mathrm{z}$ scores). Additionally, we examine the distributions of the measures of interest, transforming them to approximate normality, as required.

Hierarchical, stepwise, multiple linear regression and correlation (Cohen \& Cohen, 1975) are used to examine relationships among performance and the physiological measures described above. Each experimental hypothe- sis is stated within the framework of a proposed hierarchy of the relative contributions of independent variables to the variance of the performance metric of interest (e.g, success in discriminating vigilance targets). The entry of an independent variable into the regression equation is blocked if that portion of the dependent variable's variance has been explained by an independent variable already in the equation. Each proposed hierarchy is based on known relationships, to the extent possible. The design of the hierarchy is quite critical, since measures acquired from a single source of electrophysiological data (e.g., heart rate and systolic ejection period) may be highly correlated ("multicollinearity"). The significance of the contribution of each measure in the hierarchy to explaining the variability of the dependent variable is tested, step by step, using the $t$ statistic (Cohen \& Cohen, 1975, Model I). Measures passing this test shed light on the central neural mechanisms (by implication, based on known neural circuits) underlying performance changes. They are also assumed to be good predictors of performance success or failure. Eventually, assumed capabilities will be tested against data sets collected in similar manners to those producing the predictions. The Stepwise Regression program (P2R) in the "Biomedical" statistical package (BMDP; Dixon, 1981), plus hierarchies designed by the investigators, are used to support this statistical approach.

\section{DISCUSSION}

Attention fluctuates in a "momentary" manner, such that epoch lengths of $10 \mathrm{sec}$ to $1 \mathrm{~min}$ are probably optimal for describing physiological manifestations of changes in attention. We have used the 1-min end of this range in designing our task presentation and datacollection/reduction/analysis system to attain statistical stability in the collection of physiological data. This tactic allows adequate numbers of behavioral responses, heart periods, blinks, saccades, and 10-sec epochs of EEG to be collected in most cases. However, the tactic may also blur shorter term fluctuations in physiological manifestations of behavioral status, such as the theta activity observed in the EEGs (18-sec epochs) of truck and bus drivers on the open highway (Mackie \& Miller, 1978). Analyses of 1-min epoch data bases collected during subsequent experiments should reveal more interesting relationships. Additionally, the use of some single-subject experimental design methodologies may be appropriate.

We are most interested in how the average physiological values for each minute relate to the performance data acquired from late in that minute. This information will provide us with a context that allows the prediction of performance based upon preceding physiological activity. The measures that prove to be most reliable for explaining performance variation at this level of analysis will be flagged for further examination. Future refinements of our data-analysis capability will allow us to focus on the specific aspects of physiological activity immediately 
preceding any given stimulus. If we are able to specify the surface neuroelectrical patterns associated with good and bad performance on a long-term attention task, we should be able to provide guidance for subsequent animal, computer, or cellular-level modeling of that process. In addition, results may suggest the deletion of some independent measures, the refinement of others, and the acquisition of new measures more closely tied to the structures and processes involved in attention maintenance.

\section{REFERENCES}

Adams, O. S., Levine, R. B., \& Chiles, W. D. (1959). Research to investigate factors affecting multiple-task psychomotor performance (Tech. Rep. No. 59-120). Wright-Patterson AFB, OH: Wright Aeronautical Development Center.

BACH-Y-Rita, P., \& Collins, C. C. (Eds.). (1971). The control of eye movements. New York: Academic Press.

BARTEL, G. M. (1984). The Multiple Task Performance Battery. San Antonio, TX: OAO Corporation.

COHEN, J., \& COHEN, P. (1975). Applied multiple regression/correlation analysis for the behavioral sciences. Hillsdale, NJ: Erlbaum.

DixoN, W. J. (1981). BMDP statistical software, 1981. Berkeley, CA: University of California Press.

Gribiin, B., Steptoe, A., \& Sleight, P. (1976). Pulse wave velocity as a measure of blood pressure change. Psychophysiology, 13, 86-90.

Heather, L. W. (1969). A comparison of cardiac output values by the impedance cardiograph and dye dilution techniques in cardiac patients. In W. G. Kubicek, D. A. Witsoe, R. P. Patterson, \& A. H. L. From
(Eds.), Development and evaluation of an impedance cardiographic system to measue cardiac output and other cardiac parameters (CR101965, pp. 247-258). Houston, TX: National Aeronautics and Space Administration. (N'TIS N70-10015)

MACKIE, R. R., MILLER, J. C. (1978). Effects of hours of service, regularity of schedules, and cargo loading on truck and bus driver fatigue (TR-1765; Department of Transportation Contract DOT-HS5-01132). Goleta, CA: Human Factors Research, Inc. (NTIS PB-290-957)

Miller, J. C., \& HoRvath, S. M. (1978). Impedance cardiography. Psychophysiology, 15, 80-91.

MILLER, J. C., \& MACKIE, R. R. (1980). Vigilance research and nuclear security: Critical review and applications to security guard performance (TR-2722; National Bureau of Standards NBS-GCR-80-201). Goleta, CA: Human Factors Research, Inc.

MoRgAN, B. B., JR., \& ALLUISI, E. A. (1972). Synthetic work: Methodology for assessment of human performance. Perceptual and Motor Skills, 35, 835-845.

READER, D. C., BENEL, R. A., \& RAHE, A. J. (1981). Evaluation of a manikin psychomotor task (USAFSAM-TR-81-10). Brooks AFB, TX: USAF School of Aerospace Medicine.

StePtoe, A. (1980). Blood pressure. In I. Martin \& P. H. Venables (Eds.), Techniques in psychophysiology (pp. 247-273). New York: Wiley.

Stern, J. A., Walrath, L. C., \& Goldstein, R. (1984). The endogenous eyeblink: An indicant of cognitive activity and state. Psychophysiology, 21, 22-33.

TAKAMOTo, N. (1983). The Manikin Task. San Antonio, TX: OAO Corporation.

Williams, H. L., LUBIN, A., \& Goodnow, J. J. (1959). Impaired performance with acute sleep loss. Psychological Monographs: General and Applied, 73, $1-26$ 